\title{
Performance Comparison of Channel Allocation Techniques in Packet-Switched Mobile Communication Networks
}

\author{
Javier Gozalvez and Juan Jesús González-Delicado \\ Signal Theory and Communications Division \\ University Miguel Hernández \\ Avda de la Universidad s/n, 03202 Elche, Spain \\ j.gozalvez@umh.es
}

\begin{abstract}
Channel allocation techniques are responsible for deciding which available channel is assigned to new transmissions. Since channel quality conditions and dynamics experienced during data transmissions can be affected by the mechanism used to assign free channels, this paper proposes and evaluates different channel allocation techniques in an adaptive packet-switched mobile radio network. The common criteria used for all the techniques under consideration is to assign incoming calls the available channel that experienced the best channel quality in previous transmissions. The difference between the proposed algorithms is the metric used to evaluate the channel quality. In particular three metrics are considered: BER, BLER and mean CIR. The results obtained highlight considerable performance improvements compared to other traditional techniques, such as random channel allocation.
\end{abstract}

Keywords- channel allocation mechanism, Link Adaptation, packet switched networks

\section{INTRODUCTION}

The recent evolution of mobile communications has been characterised by the important increase in the number of subscribers, the introduction of new bandwidth-consuming multimedia services and the scarcity of the available radio resources. This evolution is being accompanied by user demands and expectations for higher Quality of Service (QoS). As a result, new challenges are faced by operators that need not only to introduce new radio interfaces but also to implement the means to efficiently manage the scarce available radio resources. The efficient use of radio resources is achieved by means of Radio Resource Management (RRM) techniques, such as channel allocation mechanisms and Link Adaptation (LA). The basis of LA is to assess the channel conditions and then use a transport mode (modulation and/or coding scheme), from a set of predefined options, that is optimised for these conditions according to a predefined criteria. The LA performance depends on the accuracy of the channel quality measurements, the ability of the system to adapt to channel quality variations and on the dynamics of the channel quality variations.

At present, most of the work that has been conducted regarding channel allocation schemes for packet data services in a GSM framework has focused on how to distribute and manage the available channels in an integrated GSM/GPRS scenario using the capacity-on demand concept and based on quality differentiation between services [1]. On the other hand, this paper focuses on different possible mechanisms to allocate a free channel (or slot in the case of TDMA systems) to a new GPRS transmission in a scenario where a given number of channels are exclusively reserved to the packet data service.

A simple and widely used mechanism to assign channels for new communications is random channel allocation. This technique chooses the channel to be assigned randomly from the unoccupied channels. The main advantages of random allocation are simplicity and the fact that all channels, and therefore equipment, are uniformly used and therefore surcharge of particular channels/equipment are avoided. Another simple proposed mechanism is sequential channel allocation. This technique assigns free channels sequentially from the start of a frame in the case of a TDMA system. The initial objective of this algorithm was to help achieving a more reliable and stable channel quality environment by reducing the variability in the number of interferers a user might experience, but at the cost of increasing the interference level for slots at the start of a frame. This stability should improve the operation of adaptive radio link techniques. However, the results presented in [2] showed that, in fact, allocation mechanisms maintaining a more constant number of interferers at the expense of a higher interference level not only decrease the throughput performance but also do not create a more stable operation of the LA algorithm.

Random and sequential slot allocation mechanisms are characterised by the fact that they don't use any specific information to assign the available channel/slots. Various other mechanisms, with different QoS objectives, have been reported in the literature. In [3], different channel allocation mechanisms are proposed to improve blocking probability whenever half and full rate connections are considered in a GSM system. Reference [4] proposes different channel allocation techniques in order to satisfy timing requirements for transmitting video frames over a GSM network. Whether considering an adaptive radio environment or using a fixed transport mode, channel allocation mechanisms can influence the channel quality conditions and dynamics and therefore the system performance. As a result, [5] proposed to exploit the flexibility in which slots can be allocated in packet-based systems to shape the interference within a system. In this

This work has been sponsored by Bancaja and the University Miguel Hernández. 
context, [6] suggested, but did not evaluate, the idea of assigning available channels based on their previously experienced channel quality as a possible mean to improve QoS. Based on this proposal, this paper proposes and evaluates three 'intelligent' channel allocation algorithms that will base their decision, on which channel to assign to incoming requests, on the channel quality previously experienced on each one of the available channels. For this purpose, three different channel quality metrics are considered: mean Carrier to Interference Ratio (CIR), mean Block Error Rate (BLER) and mean Bit Error Rate (BER). The objective of such proposals is to improve system performance in fixed and adaptive radio environments, although in this paper we will concentrate on the adaptive environment.

\section{General Packet Radio SERVICES}

This study has been conducted for packet data transmissions in a GPRS-like system. The GPRS radio interface can be modelled as a hierarchy of logical layers, each one with specific functions. This research focuses on the RLC/MAC and physical layers. While the Medium Access Control (MAC) function defines the procedures enabling multiple users to share a common transmission medium, the Radio Link Control (RLC) function is responsible for backward error correction of erroneously delivered RLC blocks by means of an ARQ protocol. The physical layer is responsible for data unit framing, data coding and the detection and correction of transmission errors by means of a FEC mechanism.

The GPRS standard defines four different coding schemes (CS); their main characteristics are shown in Table 1. These four CS offer a trade-off between throughput and coding protection, paving the way for the application of LA to GPRS.

\section{PROPOSED SLOT ALLOCATION MECHANISMS}

The three algorithms proposed in this paper base their channel assignment decision on information regarding the previously experienced channel quality in each one of the free channels at the time of the assignment.

The minBER algorithm assigns the slot that on average experienced the lower BER. This parameter was chosen since it clearly represents the channel quality conditions and also has an important relation with the QoS that a user might experience. Although, obtaining the BER on a real system is not trivial, papers such as [7] have presented methods to accurately estimate the BER. The minBLER algorithm presents the clear advantage that the BLER is an already available measure in GPRS-like systems since an acknowledgement report is regularly sent to the transmitter to indicate which packets need to be retransmitted. While the BLER measure also provides a good representation of the QoS experienced by the user, it could present the disadvantage of needing a considerable number of samples to provide an accurate and meaningful average value. The mean CIR experienced in a given burst has also been considered since it is a commonly used measure to represent the channel quality conditions. In this case, the maxCIR algorithm assigns to a new user the slot, from the available ones, that previously experienced the highest
TABLE I. GPRS CHANNEL CODING PARAMETERS

\begin{tabular}{cccc}
\hline Scheme & Code rate & Payload & Data rate (kbits/s) \\
\hline CS1 & $1 / 2$ & 181 & 9.05 \\
CS2 & $\approx 2 / 3$ & 268 & 13.4 \\
CS3 & $\approx 3 / 4$ & 312 & 15.6 \\
CS4 & 1 & 428 & 21.4 \\
\hline
\end{tabular}

CIR conditions. As with the BER, it has been shown that it is possible to quickly provide an accurate estimate of the experienced CIR [8]. However, extracting BER and CIR estimates in current systems would have an implementation cost not present in the case of BLER measures.

All the proposed algorithms operate in a similar fashion. Each slot is being provided with an array where the estimated metric (i.e. BER, BLER or CIR) for all previous transmissions will be stored. Once the array has been filled, the oldest metric estimation will be discarded. While BER and CIR values are stored for each transmission burst, BLER values (i.e. 1 or 0 depending on whether an RLC block has been received in error or not) are only stored for each RLC block transmitted. An important parameter for all algorithms, that is studied in this work, is the actual array size. When a channel has to be assigned for a new communication, the algorithm uses the metric measurements stored on the arrays. The measurements are filtered in order to get a channel quality estimate for each one of the available slots; for this paper, a filter with a rectangular shape has been initially considered. The estimation obtained will be used to decide which slot to assign.

\section{SIMULATION ENVIRONMENT}

The performance evaluation of a cellular system is usually conducted at two different levels: system level and link level. While the former models a mobile radio network, the later models the radio link at the bit level. Interfaces between both levels are hence necessary to study the overall performance.

\section{A. System Level}

The system level analysis has been conducted using an event-driven simulator working at the burst level [9]. The timescale resolution considered ensures a high modelling accuracy and allows accounting for sudden channel quality variations. The system level simulator models the channel quality in terms of the CIR and considers the first and second tier of co-channel interferers. The pathloss is predicted using the Okumura-Hata model. The shadowing has a log normal distribution with a standard deviation of $6 \mathrm{~dB}$ and a decorrelation distance of 20 meters. Fast fading has also been included by means of the link-to-system level interfaces described in the next section. Power Control or Slow Frequency Hopping mechanisms have not been implemented since they both interact with the operation of LA and the study of such interaction is not within the scope of this work.

The simulation tool, which concentrates on the downlink performance, models a cellular network of equally sized 3- 
sector macro cells, with a cluster size equal to four. Each cell has a radius of $1 \mathrm{~km}$ and each sector has been assigned two carriers (i.e. 16 channels or time slots). Although mobility has been implemented, handover between sectors has not been considered. The boundary effects have been removed by using a wrap-around technique.

Two different traffic sources have been implemented, WWW browsing and email, with the traffic type evenly distributed among users at $50 \%$. No channel partition has been applied between the two services. Users are assigned channels in a first-come-first-served basis and the channel is kept until all its data has been correctly transmitted; for this work, only single slot transmissions have been considered. To guarantee the correct transmission of the data, an ARQ protocol has been implemented following the GPRS specifications. A perfect feedback of the ARQ report with no RLC block loses has been assumed.

\section{B. Link-to-System Level Interfaces}

In order to reduce the complexity of system level simulations, the effects at the physical layer are generally included by means of Look-Up Tables (LUTs). Following the indications provided in [10], an advanced link-to-system level interface working at the burst level has been considered.

This interface is composed of two sets of LUTs. The interface requires as input from the system level the mean CIR experienced in a given burst. LUT-1 extracts the burst quality for the measured CIR. The burst quality is represented by means of the BER. LUT-1 represents a cumulative distribution function (cdf) of the BER for a given CIR. A random process is then used to generate the actual BER from the corresponding cdf. The purpose of this procedure is to model the effect of fast fading on the BER through a random process thereby including the fast fading at the system level. The BER is then estimated for the four bursts used to transmit a RLC block and LUT-2 maps the mean BER and the standard deviation of the BER over the four bursts to a corresponding BLER value. Graphical examples of LUT-1 and LUT-2 can be found in [9].

\section{Link Adaptation Algorithm}

The basis of LA is to assess the channel conditions and then use a transport mode that is optimised for these conditions. For GPRS, the adaptation will be done at the CS level as the standard considers a single modulation scheme. Since this work is based on non-real time data services, a CS is considered to be optimum if it maximises the throughput, defined as follows:

$$
\text { Throughput }=\mathrm{R}_{\mathrm{CS}} \times\left(1-\mathrm{BLER}_{\mathrm{CS}}\right)
$$

$\mathrm{R}_{\mathrm{CS}}$ and $B L E R_{\mathrm{CS}}$ are the data rate and BLER for a given CS.

The LA algorithm uses the quality measurements over the previous LA updating period to decide on the optimum CS; this paper considers a $100 \mathrm{~ms}$ LA updating period. For that purpose, the channel quality measurements are filtered using a filter with a rectangular shape. In order to decide which CS is the optimum one, the LA algorithm needs to establish the LA switching thresholds. These thresholds define the boundaries between the regions where each CS maximises the throughput.

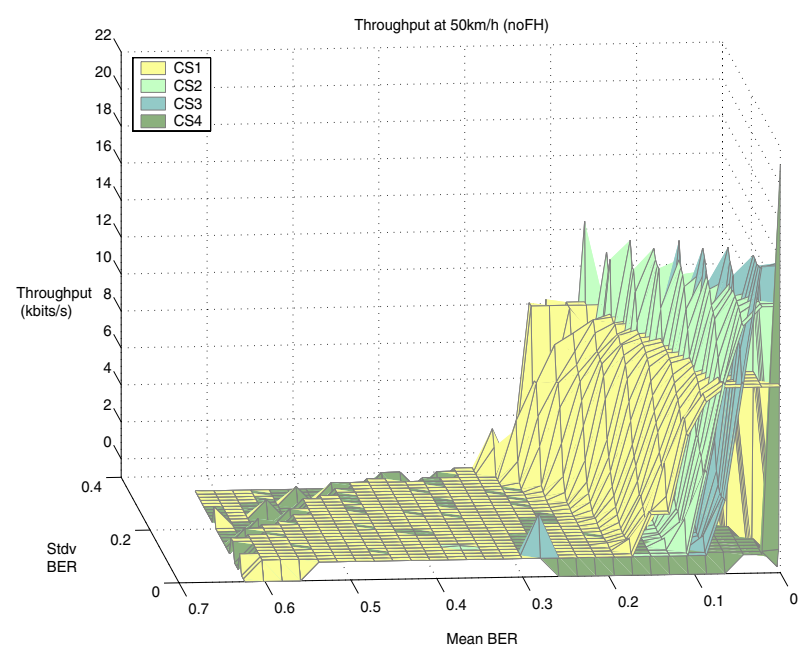

Figure 1.Throughput performance and LA switching thresholds

Since the throughput is defined as a function of the BLER, the representation of the LA switching thresholds depends on the particular link-to-system level interface considered. As illustrated in Fig. 1, the thresholds considered in this work are defined as a collection of points, each representing a combination of mean and standard deviation of burst quality values.

\section{PERFORMANCE EVALUATION}

This section evaluates the performance of the three proposed channel allocation schemes and compares it with that obtained when considering the random channel allocation scheme. This scheme has been considered the reference one because of its simplicity and its widespread adoption.

\section{A. Parameters}

Since this work is based on non-real time data services, the performance is mainly evaluated by means of the throughput and BLER. Of particular interest is not only the mean performance, but also the highest minimum performance guaranteed for $95 \%$ and $99 \%$ of the samples considered (these two parameters are extracted from the system performance cumulative distribution functions). In fact, these performance measures provide an indication of the fairness degree that can be obtained with the proposed algorithm since they show whether the performance is improved for the users that previously (i.e. using the random allocation mechanism) experienced the worst quality. When considering a LA algorithm, other interesting performance metrics are the proportion of RLC blocks received with an optimal CS and the number of CS changes per second. This last metric not only represents the signaling load associated with the use of LA but also provides an indication of the actual operation of the LA algorithm; a high number of CS changes per second could indicate that the LA algorithm is not adapting correctly to the operating conditions. Another important parameter for this study is the actual channel occupancy. Such parameter will allow us to check whether, on the long term, the proposed algorithms also distributed uniformly the channels among 
users, therefore enjoying the actual initial advantage of the random allocation mechanism.

\section{B. Performance Results}

Figure 2 illustrates the minimum throughput experienced by $95 \%$ of the samples considering the three proposed channel allocation mechanisms and the random allocation scheme. The throughput is shown as a function of the window size of the array used by each algorithm (the minBLER will get a channel quality estimate each RLC block whereas the minBER and maxCIR schemes obtain channel quality estimates each burst). First of all, it is important to note that the three proposed mechanisms clearly improve the throughput performance compared to the random allocation scheme. It can also be observed that the improvement obtained depends on the window size considered and on the particular channel allocation mechanism employed. In terms of the mechanism used, Fig. 2 shows that the maximum throughput improvement, in relation to the performance of the random allocation mechanism, is obtained with the minBLER proposal. While the BLER provides a clear indication of the quality perceived by the user, the effect that a particular BER or CIR estimate will have on the final quality depends on the CS used. This difference improves the channel assignment process of the minBLER proposal and explains its higher performance.

Fig. 2 also shows that the minBLER performance improves from the minimum window size until a window size equal to 2304 (channel quality measures), and that for larger window sizes the throughput performance does not seem to improve. The performance improvement obtained as we increase the window size is due to the fact that with larger numbers of channel quality measurements it is possible to obtain a more reliable and representative estimate of the previously experienced channel quality conditions. The results obtained have shown that using a more reliable channel quality estimate helps improving the channel allocation assignment process, i.e. it increases the probability that by assigning to a new transmission the channel that previously experienced the best channel conditions, this transmission will also experience the best possible channel quality conditions. This effect can actually be observed if we consider the usage percentage of the less robust coding scheme (CS4). While this percentage is equal to $74.4 \%$ when considering the minBLER algorithm and a window size equal to 20 , its value increases to $76.95 \%$ if the window size is set equal to 2304. Since the usage of CS4 increases when the channel conditions improve, this result shows that with the longer window sizes, the channel quality conditions actually improve. However, as illustrated in Fig.2, the improvement does not indefinitely increase with the window size. Taking into account that the actual implementation cost of the proposed schemes compared to the random allocation mechanism is mainly the memory needed to store the channel quality measurements, it seems clear that there is no interest in increasing the window size further than 2304 for the minBLER algorithm. While similar observations can be made for the maxCIR scheme and a window size equal to 2592 , the minBER algorithm seems to provide a better performance for a 5184 window size. Similar effects to that observed for the minimum throughput for $95 \%$ of the samples

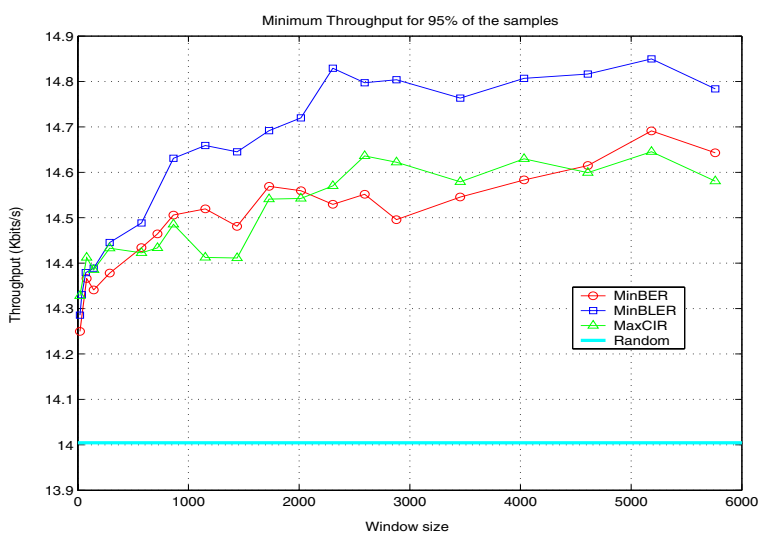

Figure 2. Minimum throughput for $95 \%$ of the samples

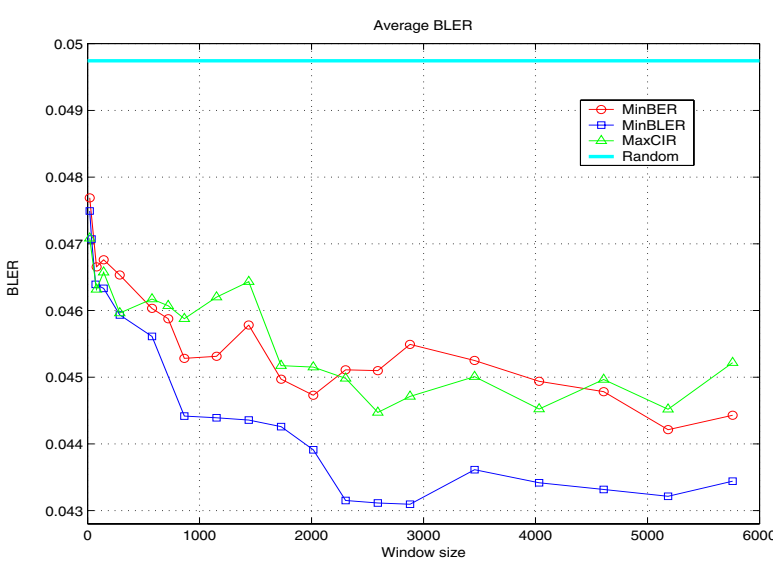

Figure 3. Average BLER

have also been obtained for the average throughput and the minimum throughput experienced for $99 \%$ of the samples. Table 2 illustrates, for these two parameters, the improvements obtained with the proposed channel allocation schemes. The results shown in this table correspond to: a window size of 2304 for the minBLER scheme; a window size of 2592 for the maxCIR scheme; and a window size of 5184 for the minBER scheme. While the improvements are not too important for the mean throughput (over $2 \%$ in the case of the minBLER proposal), the minimum throughput for $99 \%$ of the samples is greatly improved (over $11 \%$ with the minBLER proposal). These results illustrate that our proposals mainly benefit the QoS of the users that experience the worst performance.

The better throughput performance obtained with the minBLER algorithm is due to a lower average BLER (see Fig. 3). As it can be observed from Fig. 3, the improvements for the minBLER algorithm, compared to the random allocation mechanism, can exceed $10 \%$.

As illustrated in Table 2, the three proposed channel allocation schemes also have a positive effect on the actual operation of the LA algorithm. Such improvement is translated into a higher proportion of RLC blocks received with the optimal CS and an important lower average number of CS changes per second. 
TABLE II.

PERFORMANCE OF THE PROPOSED TECHNIQUES

\begin{tabular}{ccccc}
\hline & Random & MinBLER & MinBER & MaxCIR \\
\hline $\begin{array}{c}\text { Mean throughput } \\
\text { (kbits/s) }\end{array}$ & 18.76 & 19.14 & 19.08 & 19.06 \\
$\begin{array}{c}\text { Minimum throughput } \\
\text { for 99\% of samples } \\
\text { (kbits/s) }\end{array}$ & 10.88 & 12.09 & 11.85 & 11.75 \\
$\begin{array}{c}\text { Optimal CS (\%) } \\
\text { Nb of CS changes per } \\
\text { second }\end{array}$ & 75.86 & 78.56 & 78.14 & 78 \\
\hline
\end{tabular}

\section{Channel Occcupancy}

One of the main advantages of the random channel allocation mechanism, apart from its simplicity, is the fact that on the long term all channels will be uniformly used. This behavior avoids surcharging particular channels and radio equipments. As a result, it is important to check whether this feature is maintained or not with the three proposed channel allocation schemes. Figure 4 shows the average channel occupancy of each channel for the three proposed algorithms (again the window sizes considered for each algorithm correspond to the ones used in Table 2). This figure corresponds to the average occupancy across all cells modeled in our system. However, since all cells experience the same operating conditions (i.e. number of interfering cells, user load, traffic characteristics, etc), on the long term, the results obtained in a single cell will tend towards the average across all different cells. Fig. 4 clearly demonstrates that the proposed mechanisms exhibit the same channel occupancy pattern as the random channel allocation technique.

The difference between the proposed algorithms and the random channel allocation scheme is not the long term channel occupancy but the short term one. Using the three proposed techniques, it has been observed during our simulations that interfering cells avoid using the same channels at the same moment. This way our proposals guarantee a lower instantaneous interference level compared to the random channel allocation mechanism. This lower interference level is at the origin of the higher performance obtained with the three

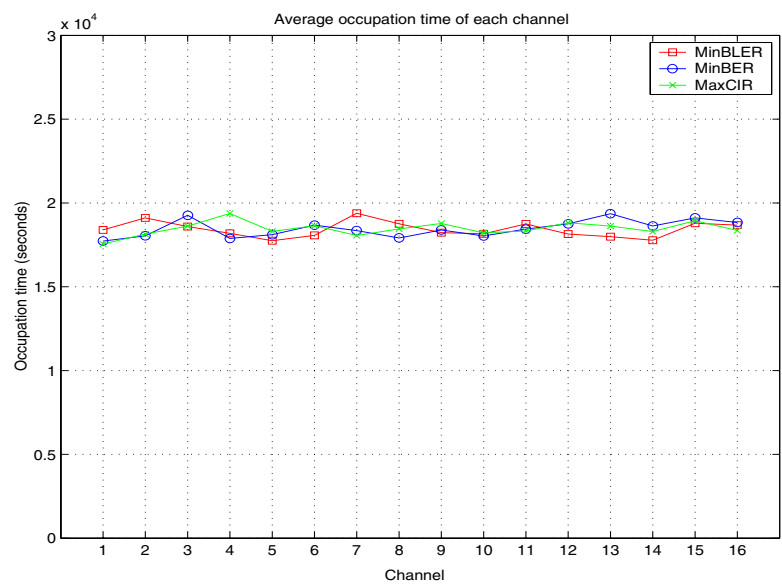

Figure 4.Channel occupancy proposed mechanisms. Again, of the three proposals, the minBLER scheme is the one guaranteeing the lowest interfering levels.

\section{CONCLUSION}

This paper has presented and evaluated three simple but 'intelligent' channel allocation mechanisms for improving the system performance of an adaptive mobile packet-switched network. The performance of the three proposed mechanisms has been compared to that obtained using the simple and commonly used random channel allocation mechanism. The results obtained demonstrate that the proposed mechanisms improve the system performance and the operation of Link Adaptation. While the minBLER algorithm has obtained the best results, the performance of all the proposals has been shown to be very sensitive to the number of channel quality measures used during the assignment process. This research has also highlighted that there is an optimum number of measures that maximizes the system performance and minimizes the cost of implementing the proposed mechanisms in a real system. Moreover, this study has demonstrated that the proposed channel allocation techniques exhibit a long term channel occupancy pattern similar to the one obtained considering the random allocation scheme. It can therefore be concluded that the proposals, while offering the benefits of the random allocation mechanism, also improve the system performance at a very low cost.

\section{REFERENCES}

[1] P. Lin and Y-B. Lin, "Channel allocation for GPRS", IEEE Transactions on Vehicular Technology, vol 50, No 2, pp 375-387, March 2001.

[2] J. Gozalvez and J. Dunlop, "Effect of slot allocation mechanisms on the performance of Link Adaptation”, Proc. of the 5th EPMCC pp 286-290, April 2003, Glasgow.

[3] M. Ivanovich, M. Zukerman, P. Fitzpatrick and M. Gitlits, "Performance between circuit allocation schemes for half- and full-rate connections in GSM", IEEE Transactions on Vehicular Technology, Vol. 47, Issue 3, pp 790-797, August 1998.

[4] Y.F. Leung, "Adaptive channel allocation methods for distributed MPEG player system over a cellular radio network", 6th International Conference on RTCSA, pp 220 - 223, December 1999.

[5] A. Arregui and J. Dunlop, "Shaping the interference distribution in a TDMA radio interface", Electronics Letters, Vol. 33, No. 3, January 1997, pp 187-188.

[6] J. Dunlop, J. Irvine, D. Robertson, and P. Cosimini, "Performance of a statistically multiplexed access mechanism for a TDMA radio interface", IEEE Personal Communications Magazine, pp. 56-64, June 1995.

[7] J. Pons and J. Dunlop, "In-service link quality estimation for Link Adaptation algorithms applied to GSM", Proc. of the IEEE ICUPC, pp. 1169-1174, October 1998, Florence.

[8] M. Andersin, N.B. Mandayam, and R.D. Yates, "Subspace based estimation of the signal to interference ratio for TDMA cellular systems", Proc. of the IEEE VTC, pp. 1155-1159, April 1996, Atlanta.

[9] J. Gozalvez and J. Dunlop, "On the dynamics of Link Adaptation updating periods for packet switched systems", Proc. of the $4^{\text {th }}$ WPMC, pp 609-614, September 2001, Aalborg.

[10] J. Gozalvez and J. Dunlop, "Link level modelling techniques for analysing the configuration of Link Adaptation algorithms in mobile radio networks", Proc. of European Wireless 2004, pp 325-330, February 2004, Barcelona. 